\title{
COMPARISON AND LINEARIZED OSCILLATION THEOREMS FOR A NONLINEAR PARTIAL DIFFERENCE EQUATION
}

\author{
B. G. ZHANG ${ }^{1}$ and JIAN-SHE YU ${ }^{2}$
}

(Received 14 March 1997)

\begin{abstract}
Connections between a linear partial difference equation with constant coefficients and a nonlinear partial difference equation are established by means of a comparison theorem and a continuous dependence of parameters theorem. A linearized oscillation theorem is also established as an application.
\end{abstract}

\section{Introduction}

Partial difference equations may be found in many branches of mathematics. Lagrange and Laplace both discussed these equations in relation to dynamics and probability, respectively. Courant et al. [2] have considered the partial difference equations of mathematical physics. In recent years, there have been rapid advances made in the solving of nonlinear problems, some of which can be modeled by nonlinear partial difference equations (see for example Agarwal [1]). Progress in this field has, in part, been due to a synergetic approach consisting of the simultaneous use of conventional analysis and numerical simulations. In [4-8], linear partial difference equations of the form

$$
x_{m+1, n}+x_{m, n+1}-p x_{m n}+q x_{m-\sigma, n-\tau}=0, \quad(m, n) \in Z^{2},
$$

where $Z^{2}=\{(m, n) \mid m, n=0,1,2, \ldots\}$, have been investigated and various properties related to the oscillatory nature of their solutions have been reported. The purpose of this paper is to establish some connections between (1) and a more general nonlinear functional inequality of the form

$$
x_{m+1, n}+x_{m, n+1}-p x_{m n}+q_{m n} f\left(x_{m-\sigma, n-\tau}\right) \leq 0, \quad(m, n) \in Z^{2},
$$

\footnotetext{
'Department of Mathematics, Ocean University of Qingdao, Qingdao 266003, P. R. China. E-mail: bgzhang@public.gd.sd.cn

${ }^{2}$ Department of Applied Mathematics, Hunan University, Changsha, Hunan 410082, P. R. China

(C) Australian Mathematical Society 2001, Serial-fee code 0334-2700/01
} 
and to use these connections to prove a linearized oscillation theorem for the associated nonlinear partial difference equation

$$
x_{m+1, n}+x_{m, n+1}-p x_{m n}+q_{m n} f\left(x_{m-\sigma, n-\tau}\right)=0, \quad(m, n) \in Z^{2} .
$$

In (2) and (3), the numbers $p, \sigma, \tau$, the sequence $\left\{q_{m n}\right\}$ and the function $f$ will be restricted by appropriate conditions. For now, we will assume throughout the rest of our paper that $p$ is a positive number, $\sigma$ and $\tau$ nonnegative integers such that $\min (\sigma, \tau)>0,\left\{q_{m n}\right\}_{(m, n) \in Z^{2}}$ a real double sequence, and $f$ a real-valued function defined on $R$. By a solution of (2) or (3), we mean a real double sequence $x=\left\{x_{m n} \mid\right.$ $m \geq-\sigma, n \geq-\tau$ \} which satisfies (2). It is not difficult to formulate and prove an existence theorem for the solutions of (2) when appropriate initial conditions are given (for example see [8]). As is customary, we say that a solution $x=\left\{x_{m n}\right\}$ of (2) is eventually positive (eventually negative) if $x_{m n}>0$ (respectively $x_{m n}<0$ ) for all large $m$ and all large $n$, and is oscillatory if it is neither eventually positive nor eventually negative.

In the next section, we will establish a comparison theorem for the relation (2). In Section 3, a continuous dependence of parameters theorem will be established for the linear difference equation (1), and relations between (3) and (1) are then obtained. A linearized oscillation theorem is given in the final section as an application.

\section{A comparison theorem}

Let $x=\left\{x_{m n}\right\}$ be an eventually positive solution of (2) such that $x_{m n}>0$ for $m \geq M-\sigma \geq 0$ and $n \geq N-\tau \geq 0$. Suppose further that $f(t)>0$ for $t>0$. Then summing (2) with respect to the second independent variable from $n$ to $\infty$, we obtain

$$
\sum_{j=n}^{\infty} x_{m+1, j}+(1-p) \sum_{j=n}^{\infty} x_{m, j+1}+p \sum_{j=n}^{\infty}\left(x_{m, j+1}-x_{m, j}\right)+\sum_{j=n}^{\infty} q_{m j} f\left(x_{m-\sigma, j-\imath}\right) \leq 0,
$$

so that

$$
\begin{aligned}
& \sum_{j=n+1}^{\infty} x_{m+1, j}+p\left(x_{m+1, n}-x_{m n}\right)+(1-p) x_{m+1, n} \\
& \quad+(1-p) \sum_{j=n}^{\infty} x_{m, j+1}+\sum_{j=n}^{\infty} q_{m j} f\left(x_{m-\sigma, j-\tau}\right) \leq 0 .
\end{aligned}
$$

Summing the above inequality with respect to the first independent variable from $m$ to $\infty$, we obtain

$$
\sum_{(i, j)=(m, n+1)}^{\infty} x_{i+1, j}+\sum_{(i, j)=(m, n)}^{\infty} q_{i j} f\left(x_{i-\sigma, j-\tau}\right)
$$




$$
+(1-p)\left\{\sum_{i=m}^{\infty} x_{i+1, n}+\sum_{(i, j)=(m, n)}^{\infty} x_{i, j+1}\right\} \leq p x_{m n} .
$$

Thus

$$
\begin{aligned}
x_{m n} \geq & \frac{1}{p}\left\{\sum_{(i, j)=(m, n+1)}^{\infty} x_{i+1, j}+\sum_{(i, j)=(m, n)}^{\infty} q_{i j} f\left(x_{i-\sigma, j-\tau}\right)\right\} \\
& +\frac{1-p}{p}\left\{\sum_{i=m}^{\infty} x_{i+1, n}+\sum_{(i, j)=(m, n)}^{\infty} x_{i, j+1}\right\}
\end{aligned}
$$

for $m \geq M$ and $n \geq N$.

Let $\vec{p}$ be a real number such that $1 \geq \bar{p} \geq p$, let $\left\{\bar{q}_{m n}\right\}_{(m, n) \in Z^{2}}$ be a nonnegative sequence such that $q_{m n} \geq \bar{q}_{m n}$ for $(m, n) \in Z^{2}$ and further let $\bar{f}$ be a real and nondecreasing function defined on $R$ satisfying $\bar{f}(x) \geq f(x)$ for $x>0$. Let $\Omega$ be the set of all real double sequences of the form $y=\left\{y_{m n} \mid m \geq M-\sigma, n \geq N-\tau\right\}$. Define an operator $T: \Omega \rightarrow \Omega$ by

$$
\begin{aligned}
(T y)_{m n}= & \frac{1}{\bar{p} x_{m n}}\left\{\sum_{(i, j)=(m, n+1)}^{\infty} x_{i+1, j} y_{i+1, j}+\sum_{(i, j)=(m, n)}^{\infty} \bar{q}_{i j} \bar{f}\left(x_{i-\sigma, j-\tau} y_{i-\sigma, j-\tau}\right)\right\} \\
& +\frac{1-\bar{p}}{\bar{p}}\left\{\sum_{i=m}^{\infty} x_{i+1, n} y_{i+1, n}+\sum_{(i, j)=(m, n)}^{\infty} x_{i, j+1} y_{i, j+1}\right\}
\end{aligned}
$$

for $m \geq M$ and $n \geq N$, and

$$
(T y)_{m n}=1
$$

elsewhere. Consider the following iteration scheme: $y^{(0)} \equiv 1$ and $y^{(j+1)}=T y^{(j)}$ for $j=0,1,2, \ldots$. Clearly, in view of (4),

$$
0 \leq y_{m n}^{(j+1)} \leq y_{m n}^{(j)} \leq 1, \quad m \geq M, n \geq N, j \geq 0
$$

Thus as $j \rightarrow \infty, y^{(j)}$ converges pointwise to some nonnegative sequence $w=\left\{w_{m n}\right\}$ which satisfies

$$
\begin{aligned}
x_{m n} w_{m n}= & \frac{1}{\bar{p}}\left\{\sum_{(i, j)=(m, n+1)}^{\infty} x_{i+1, j} w_{i+1, j}+\sum_{(i, j)=(m, n)}^{\infty} \bar{q}_{i j} \tilde{f}\left(x_{i-\sigma, j-\tau} w_{i-\sigma, j-\tau}\right)\right\} \\
& +\frac{1-\bar{p}}{\bar{p}}\left\{\sum_{i=m}^{\infty} x_{i+1, n} w_{i+1, n}+\sum_{(i, j)=(m, n)}^{\infty} x_{i, j+1} w_{i, j+1}\right\}
\end{aligned}
$$


for $m \geq M$ and $n \geq N$ and $w_{m n}=1$ elsewhere. Taking differences on both sides of the above equality, we see that the double sequence $\left\{u_{m n}\right\}=\left\{x_{m n} w_{m n}\right\}$ is an eventually non-negative solution of (3). Finally, we show that $\left\{u_{m n}\right\}$ is eventually positive, provided $\bar{q}_{m n}>0$ for $m \geq M$ and $n \geq N$. To see this, suppose to the contrary that there exists a pair of integers $m^{*} \geq M$ and $n^{*} \geq N$ such that $u_{m n}>0$ for $(m, n) \in\left\{M-\sigma,-\sigma+1, \ldots, m^{*}\right\} \times\left\{N-\tau,-\tau+1, \ldots, n^{*}\right\} \backslash\left\{\left(m^{*}, n^{*}\right)\right\}$ but $u_{m^{*} n^{*}}=0$. Then in view of (5),

$$
0 \geq \sum_{(i, j)=\left(m^{*}, n^{*}+1\right)}^{\infty} u_{i+1, j}+\sum_{(i, j)=\left(m^{*}, n^{*}\right)}^{\infty} \bar{q}_{i j} \tilde{f}\left(u_{i-\sigma, j-\tau}\right),
$$

which implies $u_{i j}=0$ for $i \geq m^{*}+1$ and $j \geq n^{*}+1$, as well as

$$
\bar{q}_{i j} \bar{f}\left(u_{i-\sigma, j-\tau}\right)=0,
$$

for $i \geq m^{*}$ and $j \geq n^{*}$. This contradicts our assumptions that $\bar{q}_{m^{*} n^{*}}>0$ and $u_{m^{*}-\sigma, n^{*}-\tau}>0$.

We summarize the above conclusions as follows.

THEOREM 1. Suppose that $\bar{p}$ and $p$ are real numbers such that $1 \geq \bar{p} \geq p>0$. Suppose that $\left\{\bar{q}_{m n}\right\}$ and $\left\{q_{m n}\right\}$ are nonnegative sequences which satisfy $q_{m n} \geq \bar{q}_{m n}>0$ for all large $m$ and $n$. Suppose further that the functions $f, \bar{f}: R \rightarrow R$ satisfy $0<\bar{f}(x) \leq f(x)$ for $x>0$. If (2) has an eventually positive solution, then so does the following equation:

$$
x_{m+1, n}+x_{m, n+1}-\bar{p} x_{m n}+\bar{q}_{m n} \bar{f}\left(x_{m-\sigma, n-\tau}\right)=0, \quad(m, n) \in Z^{2} .
$$

As an immediate consequence of Theorem 1, we have the following connection between the relation (2) and the partial difference equation (3).

COROLlaRY 1. Suppose $0<p \leq 1,\left\{q_{m n}\right\}$ is eventually positive and $f$ is positive and nondecreasing for, $x>0$. Then (2) has an eventually positive solution if, and only if, (3) has an eventually positive solution.

\section{Connections between (1) and (3)}

In order to establish the desired connections between (1) and (3), we first recall a few facts which have previously been established for (1).

A real double sequence $x=\left\{x_{m n}\right\}_{m \geq-\sigma, n \geq-\tau}$ is said to be proper if there are positive constants $M, \alpha$ and $\beta$ such that

$$
\left|x_{m n}\right| \leq M \alpha^{m} \beta^{n}
$$


for all large $m$ and $n$. The concept of proper solutions of partial difference equations is important because $Z$-transforms exist for proper solutions [3]. Indeed, by means of the method of $Z$-transform, Zhang and Liu [6] are able to derive the following result which relates the oscillatory behavior of proper solutions of (1) with the distribution of roots of an associated 'characteristic equation'.

LEMMA 1. Every proper solution of (1) oscillates if, and only if, the following characteristic equation

$$
\lambda+\mu-p+q \lambda^{-\sigma} \mu^{-\tau}=0
$$

cannot be satisfied by any pair of positive numbers $\lambda$ and $\mu$.

There are several important implications of this result. First of all, when $q \geq 0$, it is shown in [6] that every proper solution of (1) is oscillatory if, and only if,

$$
\frac{q(\sigma+\tau+1)^{\sigma+\tau+1}}{\sigma^{\sigma} \tau^{\tau} p^{\sigma+\tau+1}}>1
$$

Next, note that when $p \in(0,1]$ and $q \geq 0$, every eventually positive solution of (1) or (3) is proper. Indeed, if $x=\left\{x_{m n}\right\}$ is such a solution, then

$$
x_{m, n+1}+x_{m+1, n}-p x_{m n} \leq 0
$$

eventually, so that $x$ is eventually decreasing in $m$ and also in $n$. As a consequence, when $p \in(0,1]$ and $q \geq 0$, every solution of (1) is oscillatory if, and only if, every proper solution oscillates.

Next, note that when $q>0$, inequality (6) will still be valid when $q$ is decreased and $p$ increased by sufficiently small perturbations. Thus the following continuous dependence of parameters theorem for (1) must hold.

THEOREM 2. Suppose that $p, q>0$ and that every proper solution of (1) is oscillatory. Then there exist a nonnegative number $\xi_{1}>-p$ and a positive number $\xi_{2}<q$ such that for every $\epsilon_{1} \in\left[0, \xi_{1}\right]$ and $\epsilon_{2} \in\left[0, \xi_{2}\right]$, each proper solution of the equation

$$
x_{m+1, n}+x_{m, n+1}-\left(p+\epsilon_{1}\right) x_{m n}+\left(q-\epsilon_{2}\right) x_{m-\sigma, n-\tau}=0, \quad(m, n) \in Z^{2},
$$

is also oscillatory.

We are now ready to establish several important relations between the linear equation (1) and the nonlinear equation (3). 
THEOREM 3. Suppose $p \in(0,1]$. Suppose further that

$$
\liminf _{m, n \rightarrow \infty} q_{m n} \geq q>0 .
$$

If there is an eventually positive sequence $u=\left\{u_{m n}\right\}$ which satisfies

$$
x_{m+1, n}+x_{m, n+1}-p x_{m n}+q_{m n} x_{m-\sigma, n-\tau} \leq 0
$$

for all large $m$ and $n$, then (1) has an eventually positive solution.

PROOF. In view of (8), for any $\epsilon \in(0, q), q_{m n}>q-\epsilon$ for all large $m$ and $n$. If (9) has an eventually positive solution, then in view of Theorem 1 ,

$$
x_{m+1, n}+x_{m, n+1}-p x_{m n}+(q-\epsilon) x_{m-\sigma, n-\tau}=0
$$

also has an eventually positive solution. Therefore, if every solution of (1) is oscillatory, then by Theorem 2, there will exist an $\epsilon_{0} \in(0, q)$ such that (every proper and hence) every solution of

$$
x_{m+1, n}+x_{m, n+1}-p x_{m n}+\left(q-\epsilon_{0}\right) x_{m-\sigma, n-\tau}=0
$$

oscillates. This is the desired contradiction.

As an immediate application, suppose that $f(x) \geq x$ for $x>0$ and that (8) holds. If (3) has an eventually positive solution $u=\left\{u_{m n}\right\}$, then

and

$$
0=u_{m, n+1}+u_{m+1, n}-p u_{m n}+q_{m n} \frac{f\left(u_{m-\sigma, n-\tau}\right)}{u_{m-\sigma, n-\tau}} u_{m-\sigma, n-\tau}
$$

$$
\liminf _{m, n \rightarrow \infty} q_{m n} \frac{f\left(u_{m-\sigma, n-\tau}\right)}{u_{m-\sigma, n-\tau}} \geq \liminf _{m, n \rightarrow \infty} q_{m n} \geq q
$$

would imply, by means of Theorem 3, that (1) will have an eventually positive solution also.

THEOREM 4. Suppose that $p \in(0,1]$, that $f(x) \geq x$ for $x>0$, and that (8) holds. If (3) has an eventually positive solution, then so does (1).

Similar reasoning also leads to the following: Suppose that $p \in(0,1]$, that $(8)$ holds and that

$$
\liminf _{x \rightarrow 0^{+}} \frac{f(x)}{x} \geq 1 .
$$

If (3) has an eventually positive solution $x=\left\{x_{m n}\right\}$ which satisfies $\lim _{m, n \rightarrow \infty} x_{m n}=0$, then (1) will have an eventually positive solution. 
It is not difficult to impose conditions such that all eventually positive solutions of (3) will converge to zero as $m, n$ tend to infinity. For example, assume that

$$
\sum_{m=0}^{\infty} \sum_{n=0}^{\infty} q_{m n}=\infty
$$

For any eventually positive solution $x=\left\{x_{m n}\right\}$ of (3) where $0<p \leq 1$, since it is decreasing in $m$ and $n$ eventually, we may assume that $x$ tends to a nonnegative constant $\bar{x}$. If $\bar{x}>0$, then assuming $x_{m n}>0$ for $m \geq M-\sigma$ and $n \geq N-\tau$, we see from (4) that

$$
p x_{m n} \geq \sum_{i=m}^{\infty} \sum_{j=n}^{\infty} q_{i j} f\left(x_{i-\sigma, j-\tau}\right) .
$$

Assuming $f$ is continuous or nondecreasing on $(0, \infty)$, the infinite series of the above inequality will diverge to positive infinity, which is a contradiction. This shows that $\bar{x}=0$. Finally, note that condition (11) follows from (8). The following result is now clear.

THEOREM 5. Suppose that $p \in(0,1]$, that $(8)$ and $(10)$ hold and that $f$ is either continuous or nondecreasing on $(0, \infty)$. If $(3)$ has an eventually positive solution, then so does (1).

We now turn to the question as to when the existence of an eventually positive solution of (1) implies the existence of eventually positive solutions of (3).

THEOREM 6. Suppose that $p \in(0,1]$, that $0<q_{m n} \leq q$ for all large $m$ and $n$, and that $f(x) \leq x$ for all $x$ in a nonempty right neighborhood $(0, \delta)$ of zero. If (1) has an eventually positive solution, then so does (3).

Proof. Suppose (1) has an eventually positive solution. Then by Lemma 1 , the characteristic equation will be satisfied by a pair of positive numbers $\lambda_{0}$ and $\mu_{0}$. It is not difficult to check that the sequence $\left\{x_{m n}\right\}$ defined by $\left\{\lambda_{0}^{m} \mu_{0}^{n}\right\}$ is an eventually positive solution of (1). Furthermore, since it is easily seen from the characteristic equation that $\lambda_{0}+\mu_{0}<p \leq 1$, we see that $x_{m n} \rightarrow 0$ as $m, n$ tend to infinity. Therefore $f\left(x_{m n}\right) \leq x_{m n}$ for all large $m$ and $n$. As a consequence,

$$
\begin{aligned}
& x_{m+1, n}+x_{m, n+1}-p x_{m n}+q_{m n} f\left(x_{m-\sigma, n-\tau}\right) \\
& \quad \leq x_{m+1, n}+x_{m, n+1}-p x_{m n}+q x_{m-\sigma, n-\tau}=0
\end{aligned}
$$

for all large $m$ and $n$. We now see from Theorem 1 that (3) will have an eventually positive solution. The proof is complete. 


\section{A linearized oscillation theorem}

As is well known, it is desirable to find relatively simple equations which may serve as minorant or majorant equations for yielding qualitative properties of a given equation. In particular, in oscillation theory, it is desirable to show that a nonlinear equation, when appropriate conditions are imposed, has the same oscillatory behavior as an associated linear equation. An example of such a phenomenon, in view of our previously established results, is not difficult to illustrate. Indeed, the following result follows directly from Theorems 5 and 6 in the last section.

THEOREM 7. Suppose that $p \in(0,1]$, that $q>0$, that $\sigma, \tau$ are nonnegative integers such that $\min (\sigma, \tau)>0$ and that $f: R \rightarrow R$ is either continuous or nondecreasing on $(0, \infty)$. Suppose further that $0<f(x) \leq x$ for all $x$ in a (nonempty) right neighborhood $(0, \delta)$ of zero and that $\liminf _{x \rightarrow 0^{+}} f(x) / x=1$. Then

$$
x_{m+1, n}+x_{m, n+1}-p x_{m n}+q x_{m-\sigma, n-\tau}=0, \quad m, n=0,1,2, \ldots,
$$

has an eventually positive solution if, and only if,

$$
x_{m+1, n}+x_{m, n+1}-p x_{m n}+q f\left(x_{m-\sigma, n-r}\right)=0, \quad m, n=0,1,2, \ldots,
$$

has an eventually positive solution.

Each of the previous results related to (2) and (3) has a dual statement valid for eventually negative solutions. This is clear from the fact that $\left\{x_{m n}\right\}$ is a solution of (3) if, and only if, $\left\{-x_{m n}\right\}$ is a solution of

$$
y_{m+1, n}+y_{m, n+1}-p y_{m n}+q_{m n} F\left(y_{m-\sigma, n-\tau}\right) \geq 0, \quad m, n=0,1,2, \ldots,
$$

where $F(t)=-f(-t)$ for $t \in R$. Note that $\operatorname{sgn} F(t)=\operatorname{sgn} t$ for $t<0$, and $F$ is nondecreasing on $(0, \infty)$ when $f$ is nondecreasing on $(0, \infty)$. Thus, if in the above theorem, we assume several additional dual conditions, then we may conclude that every solution of (12) oscillates if, and only if, every solution of (13) oscillates.

THEOREM 8. Suppose that $p \in(0,1]$, that $q>0$, that $\sigma, \tau$ are nonnegative integers such that $\min (\sigma, \tau)>0$ and that $f: R \rightarrow R$ is either continuous or nondecreasing on $(-\infty, \infty) \backslash\{0\}$. Suppose further that $x f(x)>0$ for all $x \neq 0$ and $0<f(x) / x \leq 1$ in a (nonempty) deleted neighborhood $(-\delta, \delta) \backslash\{0\}$ and that $\liminf _{x \rightarrow 0} f(x) / x=1$. Then every solution of (12) oscillates if, and only if, every solution of (13) oscillates.

As an example, consider the partial difference equation

$$
x_{m+1, n}+x_{m, n+1}-p x_{m n}+q \frac{x_{m-\sigma, n-\tau}}{1+x_{m-\sigma, n-\tau}^{2}}=0, \quad m, n=0,1,2, \ldots,
$$


where $p \in(0,1], q>0$ and $\sigma, \tau$ are nonnegative integers. By Theorem 7 and its following remarks, we see that every solution of this equation oscillates if, and only if, every solution of (12) oscillates. In view of Lemma 1 , we see further that every solution of this equation oscillates if, and only if, $q(\sigma+\tau+1)^{\sigma+\tau+1}>\sigma^{\sigma} \tau^{\tau} p^{\sigma+\tau+1}$.

REMARK. Theorem 8 for $p=1$ has been obtained in [9].

\section{Acknowledgements}

Both authors were supported for this work by the NNSF of China.

\section{References}

[1] R. P. Agarwal, Difference Equations and Inequalities (Marcel Dekker, New York, 1992).

[2] R. Courant, K. Friedrichs and H. Lewy, "On partial difference equations of mathematical physics", IBM J. 11 (1967) 215-234.

[3] J. Gregor, "The multidimensional $z$-transform and its use in solution of partial difference equations", Kybernetika Supplement 24 (1988) 1-40.

[4] H. Levy and F. Lessaman, Finite Difference Equations (Dover Publications, New York, 1992).

[5] B. G. Zhang and S. T. Liu, "Oscillation of partial difference equations", PanAmerican Math. J. 5 (1995) 61-70.

[6] B. G. Zhang and S. T. Liu, "Necessary and sufficient conditions for oscillations of partial difference equations", Dynamic of Continuous, Discrete and Impulsive Systems 3 (1997) 89-96.

[7] B. G. Zhang and S. T. Liu, "On the oscillation of two partial difference equations", J. Math. Anal. Appl. 206 (1997) 480-492.

[8] B. G. Zhang, S. T. Liu and S. S. Cheng, "Oscillation of a class of delay partial difference equations", J. Difference Eq. Appl. 1 (1995) 215-226.

[9] B. G. Zhang and J. S. Yu, "Linearized oscillation theorems for certain nonlinear delay partial difference equations", Computers Math. Applic. 35 (1998) 111-116. 